\title{
Dynamic Moral Judgments and Emotions
}

\author{
Magda Osman \\ Queen Mary University of London, London, UK \\ Email: $\underline{\text { m.osman@qmul.ac.uk }}$
}

Received 14 April 2015; accepted 19 June 2015; published 23 June 2015

Copyright (C) 2015 by author and Scientific Research Publishing Inc.

This work is licensed under the Creative Commons Attribution International License (CC BY).

http://creativecommons.org/licenses/by/4.0/

(c) () D Den Access

\begin{abstract}
We may experience strong moral outrage when we read a news headline that describes a prohibited action, but when we gain additional information by reading the main news story, do our emotional experiences change at all, and if they do in what way do they change? In a single online study with 80 participants the aim was to examine the extent to which emotional experiences (disgust, anger) and moral judgments track changes in information about a moral scenario. The evidence from the present study suggests that we systematically adjust our moral judgments and our emotional experiences as a result of exposure to further information about the morally dubious action referred to in a moral scenario. More specifically, the way in which we adjust our moral judgments and emotions appears to be based on information signalling whether a morally dubious act is permitted or prohibited.
\end{abstract}

\section{Keywords}

Moral Dilemmas, Dynamic, Emotions, Updating, Dual-Processes

\section{Introduction}

The present study takes as its starting point the view that, as with many other cognitive faculties that we have which are adaptive and dynamic (e.g., decision-making, see Osman, 2010, 2014 for review), moral judgments and emotional experiences of moral dilemmas may also have similar properties (Pessoa, 2008; Wiegmann, Okan, \& Nagel, 2012). Thus, the objective of the present study is to use a novel paradigm developed by Osman (2015) in which it is possible to track changes in judgment and emotions as the result of exposure to further information about the morally dubious action referred to in a moral scenario.

\subsection{Where do Emotions End and Morals Begin?}

There are a multitude of demonstrations of the influential role emotions take on moral judgment, particularly work using the negative affect paradigm in which participants are induced to experience strong negative emo- 
tions which in turn increase the severity of their moral judgments (Schnall, Haidt, Clore, \& Jordan, 2008; Seidel \& Prinz, 2013). Work such as this adds to the support of general claims that emotions are constitutively involved in the production of moral judgments and the reasons behind those judgments (Greene et al., 2001). The most popular theoretical proposals on moral judgment and emotion suggests that in actual fact there is a distinct lack of deliberative analytical thinking when making moral judgments (Greene et al., 2001; Greene, 2008; Haidt, 2001; Haidt, \& Hersh, 2001), and instead emotions are in the driving seat; in the main because people struggle to provide reasons for their judgments. At a mechanistic level then, theories of moral cognition, such as Haidt's (2001) Social intuitionist model (Haidt, 2001) and Greene's (2008) dual-process theory, set out that there are two systems that underpin moral cognition, one which is conscious and rational, and the other which is nonconscious and emotional. "The central claim of the social intuitionist model is that moral judgment is caused by quick moral intuitions and is followed (when needed) by slow, ex post facto moral reasoning.” (Haidt, 2001: p. 181). Thus, from the standpoint of the Social intuitionist model, and other similar models of moral cognition, the causal arrow is set in the direction that emotions come first, and that they are the influential factor that informs the severity of moral judgments. Reasons simply overlay the judgments with justifications that reflect social conformity to norms that are consistent with the moral judgments made.

\subsection{Challenges to the Causal Arrow: From Emotion to Judgment}

The exact connection between emotions and moral judgments still remains contested (Teper, Zhong, \& Inzlicht, 2015), and there are many models that describe the causal structure between emotions and judgments in ways other than proposed by the Social intuitionist model (Huebner, Dwyer, \& Hauser, 2009; Moll \& de OliveiraSouza, 2007; Szekely \& Miu, 2015). For instance, several recent studies have shown that rather than moral judgments resulting from emotional signals, they may well work together as a combined representational form which are used to inform the way we judge a morally dubious action as prohibited or permissive (Duke \&Bègue, 2015; Moore, Clarke, \& Kane, 2008; Szekely \& Miu, 2015). Moreover, claims that emotions cause judgment (e.g., Greene et al., 2001; Haidt, 2001) suggest that there is a distinct temporal separation between emotion and cognition, which has also been challenged (Moll \& de Oliveira-Souza, 2007; Szekely \& Miu, 2015; Teper, Inzlicht, \& Page-Gould, 2011). In fact, this type of distinction has been argued to be an over simplification of the highly complex integrated way that emotions operate in cognitive processes (Moll \& de Oliveira-Souza, 2007; Pessoa, 2008). There has also been strong criticism of the dual-process theories, on the grounds of conceptual confusion, and the weak empirical findings on which they are supported (Huebner, 2011, 2014; Teper et al., 2015).

The focus of the discussion here is to consider the empirical work examining the association between emotions and moral judgments, and the specific empirical issues that arise, of which there appear to be three main concerns. First, many experimental set ups used to show that emotions are the cause of moral judgment involves inducing an emotional response and then examining the extent to which the emotional experience transfers to a moral judgment task. This may go some way to explaining why there is strong support for the temporal ordering from emotion to judgment, and why primary visceral states are thought to motivate moral judgments and moral behavior. In other words, the fact that emotions are seen to be the driving force behind moral judgments is simply an artefact of setting up experiments that induce emotional experiences and then examining their effects on moral judgments. Second, many of the moral dilemmas that participants are presented with are artificial and often extreme (Crockett et al., 2014; Teper et al., 2015). For this reason, many have argued that there is a lack of validity in the way in which participants' moral judgments and emotional experiences are gauged because they don’t correspond to real day-to-day dilemmas people face (Huebner, 2011, 2014; Musschenga, 2009; Sauer, 2012). Finally, there is a dynamical aspect to emotions (Oatley \& Johnson-Laird, 2014; Pessoa, 2008) which has often been overlooked in the study of the link between emotions and moral judgments. This means that there are properties of emotional experiences that have not yet been investigated in connection to moral cognition. In contrast, there is evidence showing that moral judgments are adaptive, in the sense that when different versions of the same moral dilemma (e.g., the trolley problem, an often used, but artificial moral dilemma) are presented to people, they show sensitivity to the changes in the informational content of the dilemma, and critically, they adjust their moral judgments upwards or downwards accordingly (Osman, 2015; Sinnott-Armstrong, Mallon, McCoy, \& Hull, 2008; Wiegmann et al., 2012). Therefore, without exploring the ways in which emotions and moral judgments change in light of different types of information concerning a moral dilemma, researchers limit the understanding of emotions and how they operate in moral contexts, and also research thus far limits our un- 
derstanding of whether emotions change in systematic ways, and compliment rather than drive the way in which moral judgments change over time.

\subsection{Present Study}

The main aim of the present study is to provide insights into the way in which self-reported emotional experiences as well as moral judgments change in light of exposure to further information about the morally dubious action referred to in a moral scenario. In order to generate circumstances that approximate real-life situations in which people make moral judgments a similar design was used as that by Osman (2015). Osman (2015) presented participants with moral scenarios in the form of news stories. A similar design was adopted here, along with the same stimulus set. In the present study and in Osman's (2015) study participants were shown the details of moral scenarios segmented in to the headline, main story and the conclusion. The headline signalled that a morally dubious action has been carried out. Then a more detailed description was provided in the main story which provided context behind the morally dubious action and provided a rationale for why it was carried out, and this was follow by a conclusion in which the consequences of the morally dubious action were signalled (See Table 1 for examples). Half of those tested in the present study were presented with a conclusion that included a severe consequence for the perpetrator(s) of the morally dubious action, and the other half were presented with a conclusion that had no severe consequences for the perpetrator(s); this is in line with many real world situations in which news items provide a basis on which to evaluate the morally dubious action referred to. These manipulations served three purposes. First, there is evidence to suggest that moral judgments are adjusted in the light of changes to different versions of a scenario or changes in informational content (Osman, 2015; Wiegmann et al., 2012); this lends support to the idea that moral judgments are labile rather than fixed across the presentation of details of a moral scenario. The aim here is to further test this by examining the extent to which moral judgments are adjusted when presented with additional details of the same moral scenario. Second, participants were also required to give self-reported judgments of their emotional experiences with respect to anger and disgust after reading each detail (i.e. headline, main story, conclusion) of the scenario; anger and disgust are typical emotional experiences examined in connection with moral judgments (Teper et al., 2015). Thus, it is also possible to track potential changes in the intensity of emotional experiences as a result of new information concerning a moral scenario. This is a novel experimental manipulation and as yet, has not been empirically explored. To examine this, the critically manipulation in this study involves changes to the content of the conclusion. The conclusion (i.e. severe, not severe consequences) carries different implications for whether the morally dubious actions are permissible (no severe consequence conclusion) or prohibited (severe consequence conclusion) (Osman, 2015; Waldmann, Nagel, \& Wiegmann, 2012). Indeed, Osman (2015) reported that participants were sensitive to the severity of the consequences, and adjusted their moral judgments in such a way that they were more extreme when the actions were suggested to be prohibited, and adjusted downwards when the morally dubious actions were implied to be permitted.

If participants are sensitive to the consequences of moral actions, then moral judgments should be more severe for scenarios in which there are severe consequences, than when there are no severe consequences. Assuming that emotional experiences compliment rather than cause moral judgments, then similarly, the prediction tested here is that emotional experiences should also track differences in consequences, and so intensity of disgust and anger should differ for conclusions with consequences compared to those without. Finally, one other key feature of the present study was the inclusion of two types of moral scenarios; those that were based on genuine news stories reported from national newspapers in the UK, and fabricated scenarios based on those often used commonly in moral cognition research (Incest story, Dog story_-for details see Haidt, 2001). There are rarely ever direct comparisons conducted to reveal differences in moral judgments, and emotional experiences between genuine and fabricated moral scenarios. If, as some have argued, fabricated scenarios induce different behaviors to genuine scenarios (Osman, 2015), then this should be reflected in self-reported emotional experiences. Moral judgments and emotional experiences for fabricated scenario should lead to more severe judgments and emotional experiences overall because they are typically more extreme and less nuanced moral scenarios as compared to the ones typically faced in day to day situations in real life.

\section{Methods}

Participants were randomly allocated to either: No-severe-consequence $(\mathrm{N}=41)$, Severe-consequence $(\mathrm{N}=39)$ 
Table 1. Examples of fabricated and genuine moral scenarios that were included in the Experiment.

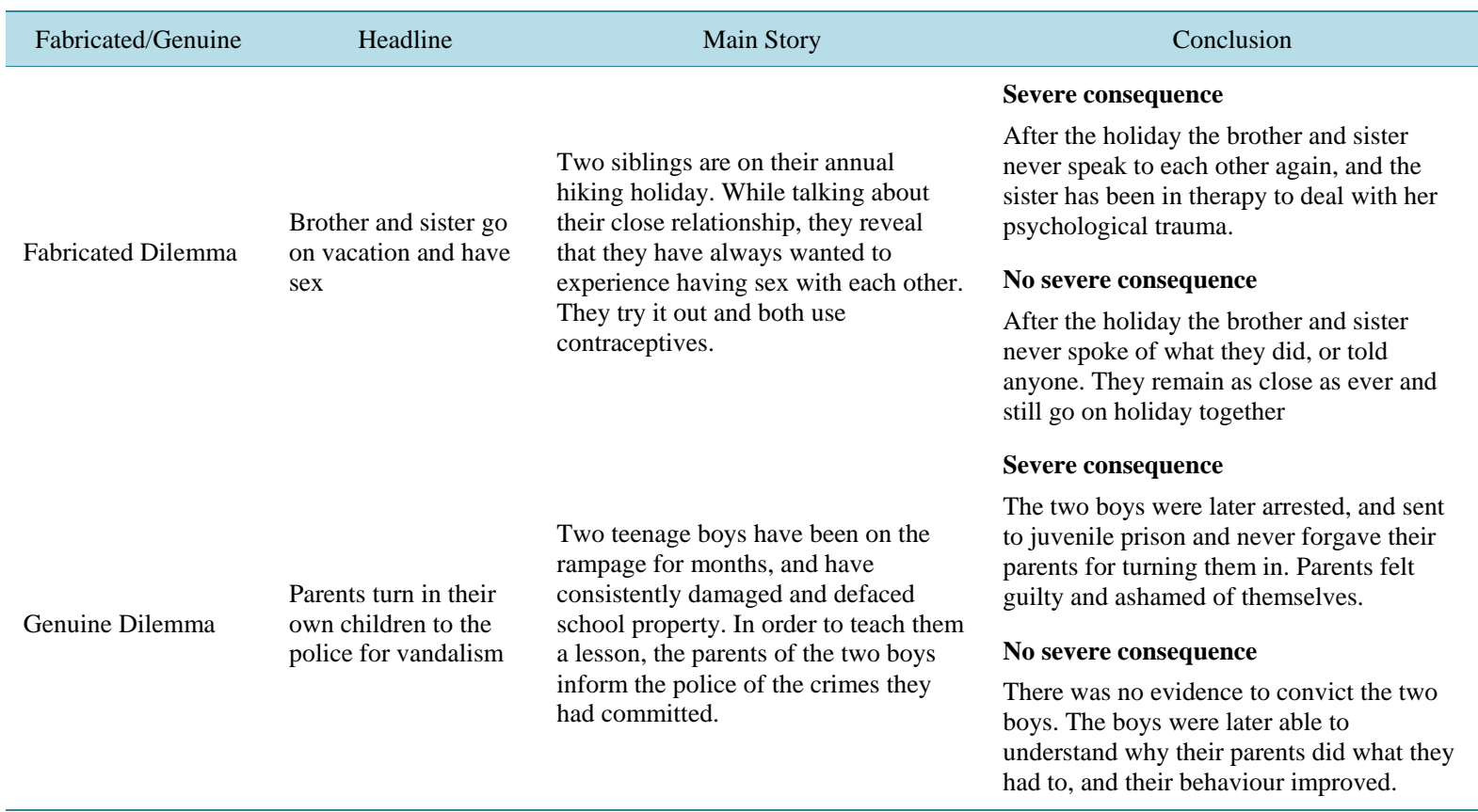

group. A total of 80 participants (19 Males) with a mean age of 21 (SD = 6.37) responded to an email advertisement to take part in the experiment. They were predominately students from across Queen Mary University of London (QMUL ethics approval register QMREC2014-15), and gave consent prior to taking part in the questionnaire.

Experiment 1 was carried out Online using Qualtrics (a web system designed to present experiments online). Participants were presented with a questionnaire comprised of six moral scenarios (See Osman, 2015 for details). They were required to make moral judgments and report their emotional experiences (disgust, anger) at their own pace for each detail concerning each of the six moral scenarios. Two of the moral scenarios were adapted from the Dog story and Incest story mentioned in the introduction section (fabricated scenarios). The four other scenarios were based on news items from UK National newspapers (broadsheets, and tabloids) over a period of three months (August 2014-October 2014).

Participants were saw a headline, a main story, and a conclusion for each of the 6 scenarios they were presented (See Table 1 ).

The order of the 6 scenarios was presented randomly to each participant, but the order of each item was fixed. Each item of the scenario was presented separately, and in the following order: The Headline was always presented first, and signalled in the simplest possible terms that the moral action that was referred to was a prohibited action. The main story included mitigating or permissible' details, and then the conclusion for half the group was presented so that it implied that the morally dubious action was prohibited (severe consequence), and for the other half the conclusion was presented so that the action was permissible (no severe consequence). The word length of the headline across scenarios ranged between 5 - 16, for the main story the word length ranged between 36 - 43, and for the conclusion the word length ranged between 23 - 34. The main manipulation comprised of randomly allocating participants to those that received all 6 moral scenarios in which a severe consequence was experienced by the perpetrator(s) (severe-consequence conclusion), and for the remaining participants, they received all 6 moral scenarios in which there was no severe consequence experienced by the perpetator(s) (no-severe consequence conclusion). For each item of the scenario (i.e. headline, main story, conclusion), participants were asked to Please indicate your moral judgment on the following scale according to what you think about the actions of the (the perpetrators were detailed here corresponding to each scenario), and responded on a scale ranging from 1 not at all morally wrong, to 7 extremely morally wrong. In addition, they also had to respond to the following: Below you can see a list of emotions, please indicate for each emotion the level of your emotional reaction based on what you have just read $(0=$ None, $1=\mathrm{A}$ little, $2=\mathrm{Some}, 3=\mathrm{A}$ lot $)$ [the 
numbers were not presented to participants only the labels]. The two emotions listed were disgust and anger. At the end of the questionnaire, participants were asked to judge for each scenario whether they thought it was fabricated or genuine, which was used as an index of participant's sensitivity to the veracity of the scenarios.

\section{Results}

\subsection{Moral judgments}

Analyses were conducted on moral judgments that were collapsed across fabricated scenarios, and separately collapsed across genuine scenarios. A 2 (type of moral scenario: fabricated, genuine) $\times 2$ (group: severe, not severe) $\times 3$ (detail: headline, main story, conclusion) ANOVA was carried out. Mauchley's test indicated that the assumption of sphericity had not been violated for type of scenario, $\chi^{2}(2)=3.6, p=.16$. But spherecity was violated for type of scenario $\mathrm{x}$ detail, $\chi^{2}(2)=22.6, p=.0002$. Because spherecity has been violated, so Greenhouse-Geisser corrected tests were reported $(\varepsilon=.8)$. In all analysis in which follow-up tests, Bonferroni correction was applied.

As observed in Figure 1, there was a clear difference such that moral judgments were more severe overall for fabricated scenarios $(M=5.5, S D=.9)$ than genuine scenarios $(M=4.2, S D=.8), F(1,78)=121.2, p<.00005$, $\eta_{p}^{2}=.61$.

It was also evident that judgments differed according to what detail a judgment was based on, $F(2,156)=33.63$, $p<.0005, \eta_{p}^{2}=.31$. Overall the headline $(M=5.2, S D=.8)$ was judged more severely than the main story ( $M$ $=4.5, S D=.8)$, and the conclusion $(M=4.3, S D=1.1)$, though no differences were found between judgments for conclusion and main stories $(t<1)$. There was an interaction between group and detail, $F(1.9,148.9)=4.88$, $p<.005, \eta_{p}^{2}=.06$. The severe consequences group judged the conclusions more severely $(M=4.8, S D=.8)$ than the no severe consequences group $(M=3.8, S D=1.1)$. In addition, the severe consequences group also judged the headline more severely $(M=5.6, S D=.8)$ than the no severe consequences group $(M=4.8, S D=.7)$, this may have been because while the order of presentation of scenarios was randomized, the order of presentation of the details of each scenario were not. This means that those presented with conclusions containing severe consequences may have harshly judged the next detail they saw for the next scenario they were presented, which was the headline from the next scenario. Thus there may have been carry over effects from the conclusion to the next headline. No other comparisons were significant $(F<1)$. Finally, there was an overall main effect, $F(1,78)$ $=20.7, p<.00005, \eta_{p}^{2}=.21$, suggesting that judgements were more severe when the consequences were severe $(M=5.2, S D=.8)$ than when they were not $(M=4.6, S D=.6)$. No other main effects or interactions were significant.

\subsection{Emotional Judgments of Disgust}

The same types of analyses conducted on moral judgments were carried out for subjective reports of disgust. Mauchley's test indicated that the assumption of sphericity was violated for type of scenario, $\chi^{2}(2)=16.3 p$ $<.0002$, so Greenhouse-Geisser corrected tests were reported $(\varepsilon=.8)$. The assumption of sphericity was not violated for type of scenario $\mathrm{x}$ detail.

As indicated in Figure 1, overall there were more extreme experiences of disgust for fabricated scenarios ( $M$ $=5.5, S D=.9)$ than genuine scenarios $(M=4.2, S D=.8), F(1,78)=263.1, p<.00,005, \eta_{p}^{2}=.77$. Also emotional experiences of disgust differed in intensity depending on what detail from the scenario was presented, $F$ $(2,156)=91.9, p<.0005, \eta_{p}^{2}=.54$. Overall there was more disgust for the headline $(M=2.8, S D=.5)$ than the main story $(M=2.5, S D=.5)$, and the conclusion $(M=2.1, S D=.6)$, and there was more disgust for the main story than the conclusion $(p<.005)$. There was an interaction such that emotional experiences of disgust differed by consequences $\mathrm{x}$ detail $\mathrm{x}$ type, $F(2,156)=7.7, p<.005, \eta_{p}^{2}=.07$. Exploring this interaction revealed that disgust was more extreme for the severe consequences group $(M=4.8, S D=.8)$ when presented with the conclusion from a genuine scenario compared with the no severe consequences group $(M=3.8, S D=1.1), F$ $(1,79)=10.5, p<.005, \eta_{p}^{2}=.08$. No other comparisons were significant $(F<1)$. No other main effects or interactions were significant.

\subsection{Emotional Judgments of Anger}

The same type of analyses conducted for judgments of disgust was conducted on judgments for anger. Mauchley's test indicated that the assumption of sphericity was violated for type of scenario, $\chi^{2}(2)=17.8$, 


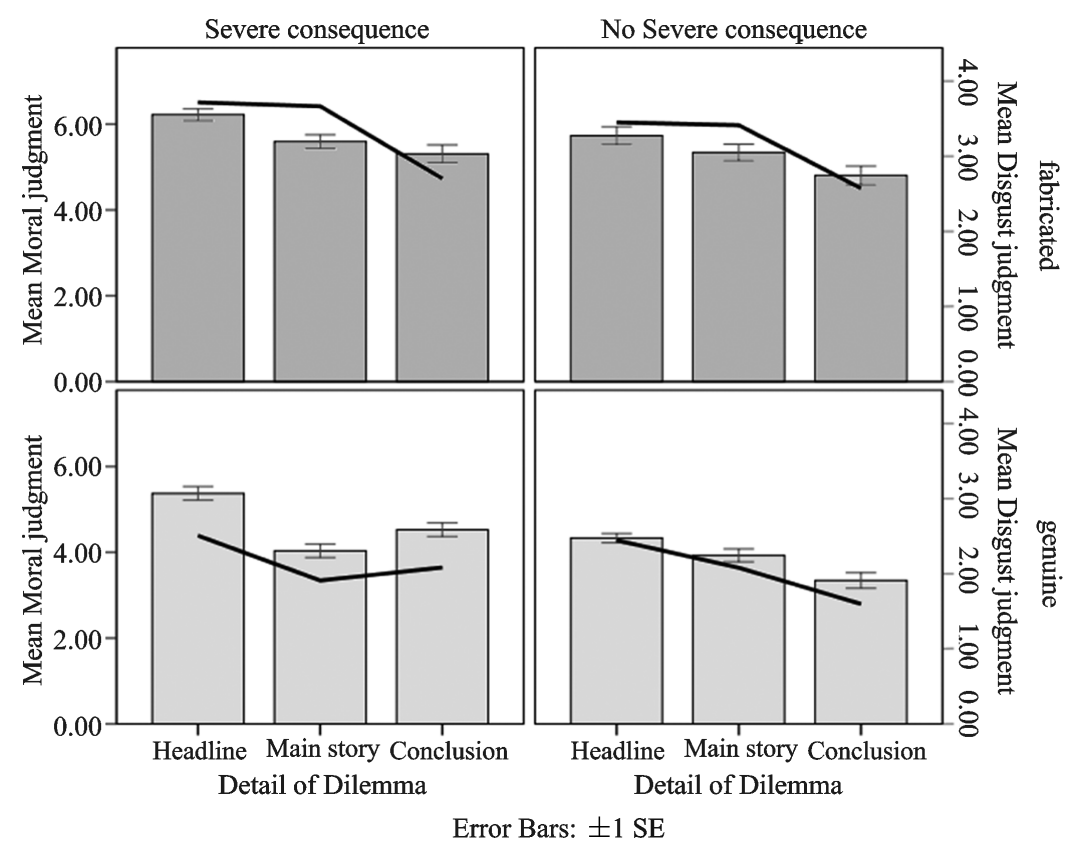

Figure 1. Mean moral judgments (bars) and disgust judgments (lines) (SE \pm 1 ) by dilemma type (fabricated, genuine) and detail (headline, main story, conclusion) and condition (No-severe-consequence, Severe-consequence).

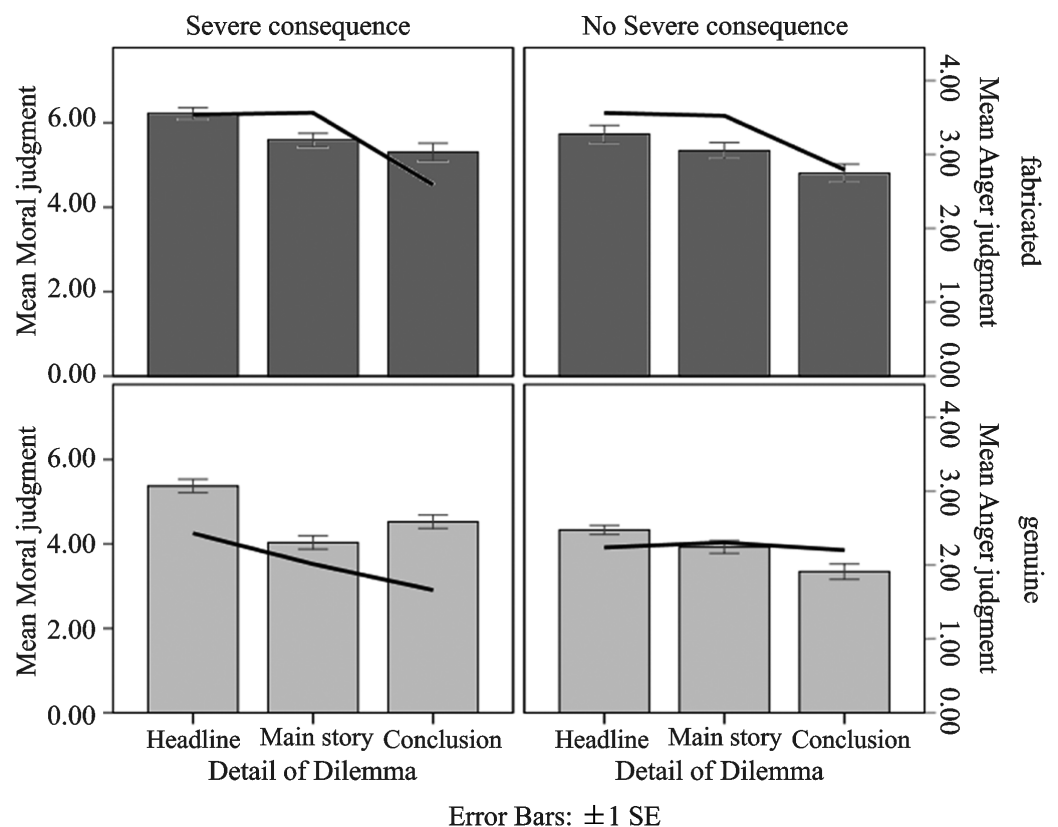

Figure 2. Mean moral judgments (bars) and anger judgments (lines) (SE \pm 1 ) by dilemma type (fabricated, genuine) and detail (headline, main story, conclusion) and condition (No-severe-consequence, Severe-consequence).

$p<.0002$, so Greenhouse-Geisser corrected tests were reported $(\varepsilon=.8)$. The assumption of sphericity was not violated for type of scenario $\mathrm{x}$ detail.

As shown in Figure 2, overall, there were more extreme experiences of anger for fabricated scenarios $(M=$ $3.3, S D=.6)$ than genuine scenarios $(M=2.1, S D=.5), F(1,78)=227.04, p<.00005, \eta_{p}^{2}=.74$.

As with disgust, anger also differed in intensity depending on what detail from the scenario was presented, $F$ $(1.6,129.7)=55.3, p<.005, \eta_{p}^{2}=.41$. Overall the conclusion led to less anger $(M=2.2, S D=.6)$ than the headline $(M=2.7, S D=.6)$, and the main story $(M=2.6, S D=.5)$, but there was no difference between the main 
story and the headline $(\mathrm{t}<1)$. There was an interaction between group and details $F(2,156)=6.2, p<.005, \eta_{p}^{2}$ $=.07$. It appears that the no severe consequences group were more angry $(M=2.4, S D=.7)$ when presented with the conclusion than the severe consequences group $(M=2.0, S D=.4)$. No other differences were significant. There was also an interaction between group $\mathrm{x}$ detail $\mathrm{x}$ type of scenario, $F(2,156)=3.1, p<.05, \eta_{p}^{2}=.04$. For genuine moral scenarios, the no severe consequence group showed more anger when presented with the conclusion $(M=2.2, S D=.7)$ than the severe consequence group $(M=1.7, S D=5)$. The same pattern was revealed for fabricated moral scenarios, again the no severe consequence group showed more anger $(M=2.3, S D$ $=.6)$ than the severe consequence group $(M=2.0, S D=7)$. No other comparisons were significant $(F<1)$. No other main effects or interactions were significant.

\subsection{Correlation between Moral Judgments and Emotions}

Moral judgments were collapsed across all six scenarios for each of the three details, and these were correlated with mean subjective reports of disgust for each of the three details collapsed across the scenarios, and similarly for subjective reports of anger. The analyses were split by group. Overall, there were no significant correlations between moral judgments and anger, therefore the rest of this section concerns the correlational analysis for moral judgments and disgust.

Severe Consequences group: A Pearson product-moment correlation coefficient analysis showed that moral judgments of headlines correlated positively with judgments of disgust when the headlines were presented ( $r(39)$ $=.38, p=.018$ ). Moral judgments of the main news item of the moral scenarios was positively correlated with self-reports of disgust $(r(39)=.52, p=.001)$, and the conclusions, $(r(39)=.42, p=.007)$. Also, there was a moderate positive correlation between moral judgments of the conclusion of moral scenarios and subjective reports of disgust associated with the headline $(r(39)=.42, p=.008)$, and the conclusion, $(r(39)=.41, p=.008)$.

No Severe Consequences group: A Pearson product-moment correlation coefficient analysis showed that moral judgments of headlines correlated positively with judgments of disgust $(r(41)=.41, p=.006)$. Moral judgments of the main news item of the moral scenarios was positively correlated with judgments of disgust associated with the headlines $(r(41)=.34, p=.02)$, judgments of disgust for the main news details $(r(41)=.67, p$ $=.00002)$, and judgements of disgust for the conclusions $(r(41)=.48, p=.002)$. No other correlations approached significance.

\subsection{Detection of Fabricated and Genuine Scenarios}

Finally, turning to detection of fabricated and genuine scenarios, a wilcoxon signed rank test revealed that participants were more accurate at judging the genuine as real $(M=41 \%, S D=30)$ than the fabricated scenarios as made-up $(M=79 \%, S D=19), Z=7.8, p<.005$. This suggests that participants mistakenly judged the fabricated scenarios as genuine more often than the genuine scenarios as fabricated.

\section{Discussion}

The present study was designed to examine whether moral judgments and emotional experiences changed in systematic ways in response to exposure to further information about the morally dubious action referred to in a scenario. To this end, the study provided new evidence that moral judgments and emotional experiences of disgust and anger are dynamic, and adjusted as more information is presented. Moreover, the study replicated the basic findings reported by Osman (2015) showing that people adjust and update their moral judgments in light of new information regarding a moral scenario, and extends this work to suggest that emotional judgments are also flexible and adjusted in response to further information regarding morally dubious actions.

\subsection{Dynamics of Moral Judgments and Emotional Experiences}

The present study showed that moral judgments changed in response to different details concerning the moral scenarios, and while participants gave the most severe judgments for the initial limited information regarding the scenario (i.e. the headline), they adjusted the severity of their judgments downwards as more information was provided (i.e. main story, conclusion). In other words, when context was provided for why a morally dubious action was carried out, people used this to inform their later judgments and consciously integrated this new information into their judgments of the action. Crucially, this reflects the fact that judgments and emotions are not 
fixed, and that they are likely to operate on rational processes (Huebner, 2011, 2014; Teper et al., 2015). More to the point, this evidence suggests that there may well be an integrated representation of the moral scenario that is based on informational content as well as personal emotional experiences that signal the valance on which the information should be judged. The evidence from the present study suggests that both moral judgments and emotional experiences change systematically in response to changes in information that critically concern the way in which a morally dubious action should be evaluated.

In addition, there appeared to be a close correspondence between moral judgments and self-reported feelings of disgust, whereas this was not the case for self-reported feelings of anger. It may well be the case that: (1) emotional experiences of disgust more closely track moral judgments over and above experiences of anger, (2) both moral judgments and experiences of disgust are more closely aligned when considering information from moral scenarios as compared to anger. The evidence from this study is not designed is such a way as to be able to empirically discriminate between these two possibilities. However, what is clear from the evidence from this study is that people's moral judgments and their emotional experiences are dynamic and change in ways that track changes in informational content regarding a moral scenario, in particular information that signals the extent to which a morally dubious action is permitted or prohibited. Critically, the findings suggest that, at least given the experimental set up used in this study, that one key driving force behind changes in moral judgments and emotional experiences are driven by changes in informational content about the morally dubious action, and so updating is guided by external factors rather than changes primarily from emotional experiences independent on the morally dubious action referred to in the scenario.

In addition, the evidence from the present study is not consistent with the view that moral cognition is supported by two separable systems (one conscious, one unconscious), given that moral judgments and emotional experiences changed in systematic and complimentary ways in response to exposure to further information about the morally dubious action referred to in a scenario. If emotions are the cause of moral judgments, and if people have limited access to, and cannot directly modify their emotional experiences because they are simple primary drivers of the way in which morally dubious actions are perceived, then one might expect little change in emotional experiences throughout the reading of moral scenarios in which moral outrage is experienced, and sustained throughout the processing of the moral scenario. This was not the case in the present study.

\subsection{Consequences Matter}

The present study revealed that consequences impacted moral judgments and emotions. Moral judgments were more severe and experiences of disgust were more intense when there was a severe consequence for the perpetrator(s) of the morally dubious action outlined in the conclusion. For anger, experiences were more intense when there were no severe consequences than when there were severe consequences. One reason for this is that while the conclusion signalled that the morally dubious action may have been permissible, which was reflected in their moral judgments and their experiences of disgust, participants may have expected a different outcome in which the transgressor was punished, and the fact the transgressor was not punished may have led to elevated experiences of anger. Consistent with this conjecture, there is some evidence to suggest that when morally dubious actions go unpunished, individuals do show intense anger (for discussion see Teper et al., 2015). It is also important to note that the nuanced experiences of emotions in response to the different consequences for the perpetrator(s) reported in the conclusions were predominately found for genuine scenarios rather than the fabricated scenarios typically used to study moral cognition in psychology, which again suggests that when it comes to real-life situations, people show a complex emotional understanding of the moral scenario. Moreover, it suggests that researchers should take care in the choice of moral scenarios that they present people, and that artificial scenarios used in the lab may not entirely reflect the types of real world processes that people engage in when they are making judgments or experience emotions concerning morally dubious actions.

\subsection{Veracity of Moral Scenarios}

Overall, the severity of moral judgments and the intensity of emotional experiences were higher for fabricated than for genuine moral scenarios. This suggests that the criticisms concerning the use of highly artificial moral scenarios may indeed be warranted (Huebner, 2011, 2014; Musschenga, 2009; Sauer, 2012; Teper et al., 2015). Also, accuracy was higher when identifying genuine rather than fabricated scenarios, though it is important to note that the accuracy of detection may reflect the tendency for participants to judgment most of the scenarios as 
genuine.

\section{Conclusion}

The present study provides new insights into the way in which moral judgments and emotional experiences of moral scenarios change in response to new information. The general implication from this study is that people adjust their judgments and emotional experiences in systematic ways based on evaluations of critical informational details from the moral scenario itself. This also points to the fact that people likely have conscious access to the basis on which they are making judgments and respond emotionally to morally dubious acts. Finally, the findings from this study strongly suggest that caution should be taken when presenting people with fabricated moral scenarios, for the reason that moral judgments and emotional experiences differed in substantive ways for genuine moral scenarios as compared to fabricated moral scenarios typically used by moral psychology researchers. Finally, the findings from this study present a challenge to the view that emotions are the key drivers of moral judgments and deliberate conscious thinking reflects post-hoc rationalisation which suggests a dualprocess conceptualisation of moral cognition. Instead, it appears that we have a complex representation of moral scenarios and use a number of sources of information (external \& internal) in order to guide the way we process information and evaluate it to form judgments about what is good and bad.

\section{References}

Crockett, M. J., Kurth-Nelson, Z., Siegel, J. Z., Dayan, P., \& Dolan, R. J. (2014). Harm to Others Outweighs Harm to Self in Moral Decision Making. Proceedings of the National Academy of Sciences, 111, 17320-17325. http://dx.doi.org/10.1073/pnas.1408988111

Greene, J. D., Sommerville, R. B., Nystrom, L. E., Darley, J. M., \& Cohen, J. D. (2001). An fMRI Investigation of Emotional Engagement in Moral Judgment. Science, 293, 2105-2108. http://dx.doi.org/10.1126/science.1062872

Greene, J. D. (2008). The Secret Joke of Kant’s Soul. In W. Sinnott-Armstrong (Ed.), Moral Psychology (Vol. 3, pp. 35-80). Cambridge, MA: MIT Press.

Haidt, J. (2001). The Emotional Dog and Its Rational Tail: A Social Intuitionist Approach to Moral Judgment. Psychological Review, 108, 814-834. http://dx.doi.org/10.1037/0033-295X.108.4.814

Haidt, J., \& Hersh, M. A. (2001). Sexual Morality: The Cultures and Reasons of Liberals and Conservatives. Journal of Applied Social Psychology, 31, 191-221. http://dx.doi.org/10.1111/j.1559-1816.2001.tb02489.x

Huebner, B., Dwyer, S., \& Hauser, M. (2009). The Role of Emotion in Moral Psychology. Trends in Cognitive Sciences, 13, 1-6. http://dx.doi.org/10.1016/j.tics.2008.09.006

Huebner, B. (2011). Critiquing Empirical Moral Psychology. Philosophy of the Social Sciences, 41, 50-83. http://dx.doi.org/10.1177/0048393110388888

Huebner, B. (2014). Do Emotions Play a Constitutive Role in Moral Cognition? Topoi, 1-14.

Moll, J., \& de Oliveira-Souza, R. (2007). Moral Judgments, Emotions and the Utilitarian Brain. Trends in Cognitive Sciences, 11, 319-321. http://dx.doi.org/10.1016/j.tics.2007.06.001

Moore, A., Clark, B., \& Kane, M. (2008). Who Shalt Not Kill? Individual Differences in Working Memory Capacity, Executive Control, and Moral Judgment. Psychological Science, 19, 549-557.

http://dx.doi.org/10.1111/j.1467-9280.2008.02122.x

Musschenga, A. W. (2009). Moral Intuitions, Moral Expertise and Moral Reasoning. Journal of Philosophy of Education, 43, 597-613. http://dx.doi.org/10.1111/j.1467-9752.2009.00707.x

Oatley, K., \& Johnson-Laird, P. N. (2014). Cognitive Approaches to Emotions. Trends in Cognitive Sciences, 18, $134-140$. http://dx.doi.org/10.1016/j.tics.2013.12.004

Osman, M. (2010). Controlling Uncertainty: A Review of Human Behavior in Complex Dynamic Environments. Psychological Bulletin, 136, 65-86. http://dx.doi.org/10.1037/a0017815

Osman, M. (2014). Future-Minded: The Psychology of Agency and Control. London: Palgrave-MacMillan.

Osman, M. (2015). Moral Judgments: Truth, Order and Consequence. Psychology, 6, 633-642. http://dx.doi.org/10.4236/psych.2015.65061

Pessoa, L. (2008). On the Relationship between Emotion and Cognition. Nature Reviews Neuroscience, 9, 148-158. http://dx.doi.org/10.1038/nrn2317

Sauer, H. (2012). Morally Irrelevant Factors: What's Left of the Dual Process-Model of Moral Cognition? Philosophical Psychology, 25, 783-811. http://dx.doi.org/10.1080/09515089.2011.631997 
Schnall, S, Haidt, J., Clore, C., \& Jordan, A. (2008). Disgust as Embodied Moral Judgment. Personality and Social Psychology, 34, 1096-1109. http://dx.doi.org/10.1177/0146167208317771

Seidel, A., \& Prinz, J. (2013). Sound Morality. Cognition, 127, 1-5. http://dx.doi.org/10.1016/j.cognition.2012.11.004

Sinnott-Armstrong, W., Mallon, R., McCoy, T., \& Hull, J. (2008). Intention, Temporal Order, and Moral Judgments. Mind \& Language, 23, 90-106. http://dx.doi.org/10.1111/j.1468-0017.2007.00330.x

Szekely, R. D., \& Miu, A. C. (2015). Incidental Emotions in Moral Dilemmas: The Influence of Emotion Regulation. Cognition and Emotion, 29, 64-75. http://dx.doi.org/10.1080/02699931.2014.895300

Teper, R., Inzlicht, M., \& Page-Gould, E. (2011). Are We More Moral than We Think? Exploring the Role of Affect in Moral Behavior and Moral Forecasting. Psychological Science, 22, 553-558. http://dx.doi.org/10.1177/0956797611402513

Teper, R., Zhong, C. B., \& Inzlicht, M. (2015). How Emotions Shape Moral Behavior: Some Answers (and Questions) for the Field of Moral Psychology. Social and Personality Psychology Compass, 9, 1-14. http://dx.doi.org/10.1111/spc3.12154

Waldmann, M. R., Nagel, J., \& Wiegmann, A. (2012). Moral Judgment. In K. J. Holyoak, \& R. G. Morrison (Eds.), The Oxford Handbook of Thinking and Reasoning (pp. 364-389). http://dx.doi.org/10.1093/oxfordhb/9780199734689.013.0019

Wiegmann, A., Okan, Y., \& Nagel, J. (2012). Order Effects in Moral Judgment. Philosophical Psychology, 25, 813-836. http://dx.doi.org/10.1080/09515089.2011.631995 